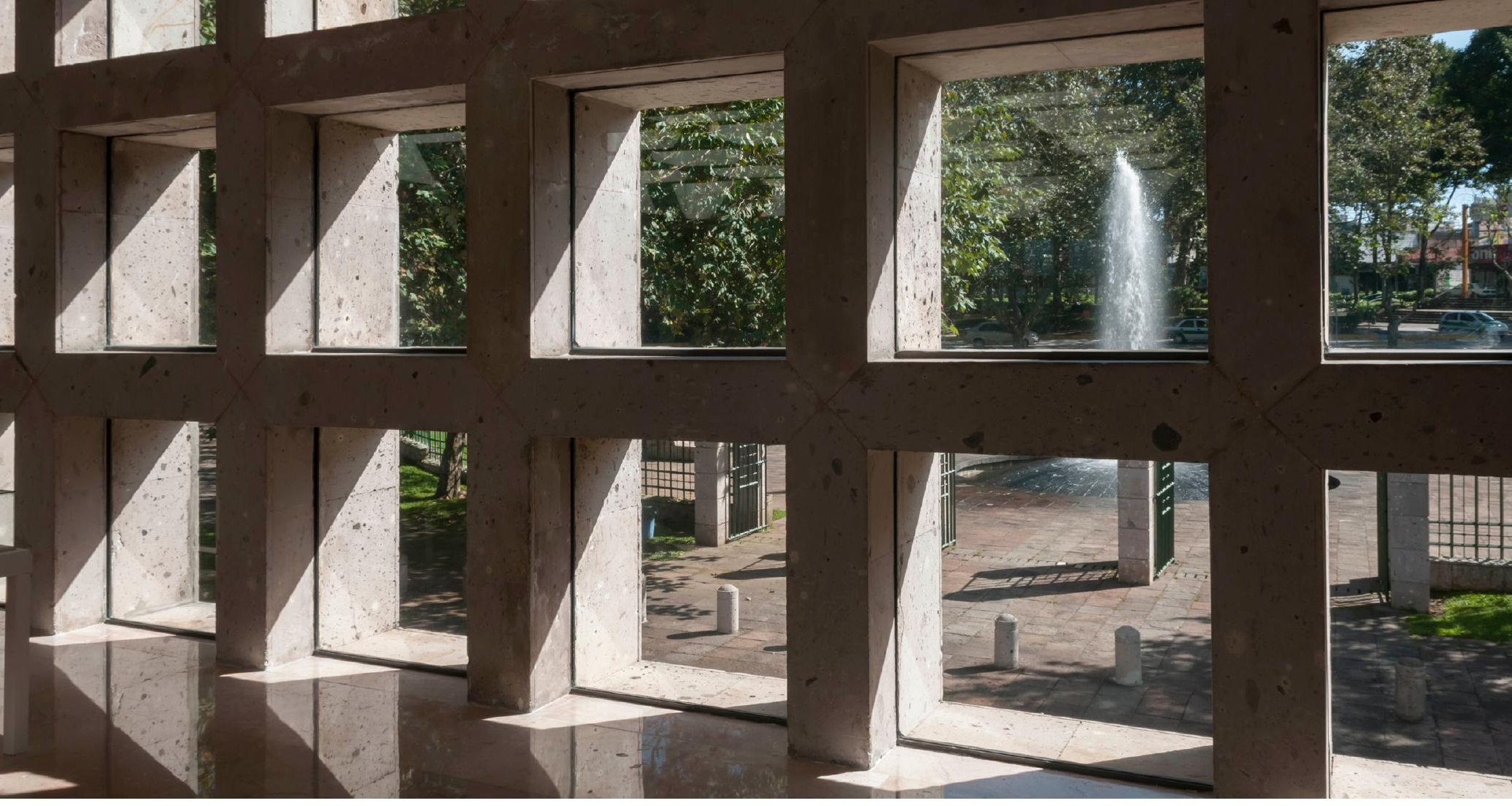

\title{
Metodología para el diagnóstico de los museos en Veracruz
}

\section{Azminda Meybelli Román Nieto ${ }^{1}$ - Maura Ordóñez Valenzuelaํ- Omar Melo Martínez ${ }^{1}$}

RESUMEN: El presente artículo describe la metodología que se implementará para el diagnóstico de los museos en Veracruz. En esta primera fase, se presenta el diseño de la herramienta metodológica para la obtención de los datos, la cual toma como referencia la información contenida en el Sistema de Información Cultural (SIC), Instituto Nacional de Estadística y Geografía (INEGI) y el Registro de Museos Iberoamericanos (RMI), y que servirán como base para la elaboración del registro estatal de museos $\mathrm{y}$, posteriormente, la cartografía respectiva, como parte de las líneas de investigación del Observatorio Universitario de Museos (OUM) en un lapso de dos años.

ABSTRACT: This article describes the methodology that will be implemented for the diagnosis of museums in Veracruz. In this first phase, the design of the methodological tool for data collection is presented, which takes as reference the information contained in the
Cultural Information System (SIC), National Institute of Statistics and Geography (INEGI) and the Registry of Ibero-American Museums (RMI), which will serve as a basis for the elaboration of the state registration of museums and later the respective cartography, as part of the research lines of the University Museum Observatory (OUM) within two years.

\section{Introducción}

El Observatorio Universitario de Museos del Museo de Antropología de Xalapa, se planteó como uno de sus ejes estratégicos el diagnóstico y monitoreo de los museos de Veracruz. Estas acciones permitirán saber cuántos museos hay en la entidad, su tipología y estado actual, con la finalidad de conocer la realidad museológica del estado, su diversidad y con ello, poder establecer parámetros de comparación con otros estados del país.

1 Museo de Antropología de Xalapa, Observatorio Universitario de Museos del Museo de Antropología de Xalapa OUM MAX,email:azroman@uv.mx;mordoñez@uv.mx; omelo@uv.mx 
Para el logro de este objetivo, hemos planteado una metodología mixta para el diagnóstico de los museos. En esta primera etapa, se diseñó una ficha de registro en la que se clasificaron los elementos que integran un museo, a su vez cada categoría contenía una subcategoría que permitirá que la información sea más específica. La recolección de la información se plantea en dos fases: 1) cotejo de las siguientes fuentes de información: Sistema de Información Cultural (SIC), Instituto Nacional de Estadística y Geografía (INEGI) y del Registro Iberoamericano de Museos (RMI), de la plataforma IBERMUSEOS; 2) Visita a los recintos. El diseño de la herramienta ${ }^{2}$ contempla los siguientes datos:

\begin{tabular}{|c|c|c|}
\hline Categoría & \multicolumn{2}{|c|}{ Subcategoría } \\
\hline \multirow{6}{*}{ Tipología } & \multicolumn{2}{|c|}{ Federal } \\
\hline & \multicolumn{2}{|c|}{ Estatal } \\
\hline & \multicolumn{2}{|c|}{ Municipal } \\
\hline & \multicolumn{2}{|c|}{ Comunitario } \\
\hline & \multicolumn{2}{|c|}{ Universitario } \\
\hline & \multicolumn{2}{|c|}{ Privado } \\
\hline \multirow{3}{*}{ Ubicación } & \multicolumn{2}{|c|}{ Municipio } \\
\hline & \multicolumn{2}{|c|}{ Localidad } \\
\hline & \multicolumn{2}{|c|}{ Dirección } \\
\hline \multirow{8}{*}{ Información general } & \multicolumn{2}{|c|}{ Costo General } \\
\hline & \multicolumn{2}{|c|}{ Costo Preferencial } \\
\hline & \multicolumn{2}{|c|}{ Horario } \\
\hline & \multicolumn{2}{|c|}{ Teléfonos } \\
\hline & \multicolumn{2}{|c|}{ Fax } \\
\hline & \multicolumn{2}{|c|}{ Página electrónica } \\
\hline & \multicolumn{2}{|c|}{ Correo electrónico } \\
\hline & \multicolumn{2}{|c|}{ Redes Sociales } \\
\hline \multirow{11}{*}{ Servicios } & \multicolumn{2}{|c|}{ Taquilla } \\
\hline & \multicolumn{2}{|c|}{ Paquetería } \\
\hline & \multicolumn{2}{|c|}{ Recorridos guiados } \\
\hline & \multicolumn{2}{|c|}{ Audio guías } \\
\hline & \multicolumn{2}{|c|}{ Accesos (rampas) } \\
\hline & \multicolumn{2}{|c|}{ Cafetería } \\
\hline & \multicolumn{2}{|c|}{ Renta de espacios } \\
\hline & \multicolumn{2}{|c|}{ Red inalámbrica } \\
\hline & \multicolumn{2}{|c|}{ Auditorio/salón de usos múltiples } \\
\hline & & \\
\hline & & \\
\hline & Fdificin & Propio \\
\hline Instalaciones & EAIIITIO & Histórico \\
\hline Mistalderites & & \\
\hline & & \\
\hline
\end{tabular}

2 Una primera versión de esta propuesta se puede consultar en: Proyecto ArKeopatías. / "Textos de la casa \#118”. México 2017. https://arkeopatias.wordpress.com/ en línea 


\begin{tabular}{|c|c|c|}
\hline Categoría & \multicolumn{2}{|c|}{ Subcategoría } \\
\hline Instalaciones & \multicolumn{2}{|c|}{ Mantenimiento } \\
\hline \multirow{3}{*}{ Historia } & \multicolumn{2}{|c|}{ Fecha de fundación } \\
\hline & \multicolumn{2}{|c|}{ Fecha de apertura al público } \\
\hline & \multicolumn{2}{|c|}{ Reaperturas } \\
\hline \multirow{6}{*}{ Colección } & \multicolumn{2}{|c|}{ Tipo de acervo } \\
\hline & \multicolumn{2}{|c|}{ Número de piezas } \\
\hline & \multicolumn{2}{|c|}{ Periodos/Temporalidades } \\
\hline & \multicolumn{2}{|c|}{ Rasgos culturales } \\
\hline & \multirow{2}{*}{ Procedencia } & Investigación \\
\hline & & Donación \\
\hline \multirow{3}{*}{ Tipo de exposición } & \multicolumn{2}{|c|}{ Permanente } \\
\hline & \multicolumn{2}{|c|}{ Temporal } \\
\hline & \multicolumn{2}{|c|}{ Itinerante } \\
\hline \multirow{4}{*}{ Experiencia Museográfica } & \multicolumn{2}{|c|}{ Contemplativa } \\
\hline & \multicolumn{2}{|c|}{ Contextual o didáctica } \\
\hline & \multicolumn{2}{|c|}{ Ambiental } \\
\hline & \multicolumn{2}{|c|}{ Interactiva } \\
\hline \multirow{6}{*}{ Servicios educativos } & \multicolumn{2}{|c|}{$\begin{array}{c}\text { Publicaciones } \\
\text { (libros, folletos, guías) }\end{array}$} \\
\hline & \multicolumn{2}{|c|}{ Talleres/cursos } \\
\hline & \multicolumn{2}{|c|}{ Recorridos temáticos } \\
\hline & \multicolumn{2}{|c|}{ Materiales didácticos y de apoyo escolar } \\
\hline & \multicolumn{2}{|c|}{ Proyección de materiales audiovisuales } \\
\hline & \multicolumn{2}{|c|}{ Eventos académicos } \\
\hline \multirow{4}{*}{$\begin{array}{l}\text { Tipo de público } \\
\text { (con base en la edad) }\end{array}$} & \multicolumn{2}{|c|}{ Infantil } \\
\hline & \multicolumn{2}{|c|}{ Juvenil } \\
\hline & & ltos \\
\hline & & a Edad \\
\hline & & ual \\
\hline Afluencia estimada & & nales \\
\hline & & njeros \\
\hline & Tine & Recorrido guiado \\
\hline Visita & 11 ро & Recorrido Libre \\
\hline & Duración & $\begin{array}{l}\text { Se establece la duración aproxi- } \\
\text { mada según cada recinto }\end{array}$ \\
\hline
\end{tabular}

Fuente: Elaboración propia

\section{Tipología}

El universo tipológico de los museos es muy amplio, su clasificación permite comprender y definir con mayor claridad sus actividades y funciones, además de establecer sus objetivos y metas, así como sus compromisos, ya sean sociales, económicos y culturales.
Existen varias formas de clasificar los recintos museísticos. Por ello, existen diversas tipologías de museos, que van a determinar las condiciones de su funcionamiento diario, para el diseño de la ficha, se utilizó la clasificación propuesta por Rodrigo Witker, (2001), la cual los cataloga de la siguiente manera: por temática y tipo de colección, por el origen de sus re- 
cursos administrativos, por su área de influencia y ubicación, por el tipo de público al cual está dirigido y por el tipo de exposición.

\section{Rasgos generales, infraestructura y servicios}

En este punto, se tomaron como referencia las nuevas perspectivas museológicas mencionadas por Witker (2001), las cuales proponen que el desarrollo de la planeación arquitectónica de los museos, debe estructurarse de una manera equitativa, armónica y funcional de acuerdo a cuatro puntos básicos en todo museo:

Áreas de administración, operación y mantenimiento: Las conforman oficinas de dirección, administración, difusión, servicios educativos, talleres de mantenimiento, plantas de aire y luz.

Áreas de investigación y almacenamiento: Son todos los talleres, laboratorios de estudio, manejo y conservación de las colecciones permanentes y temporales, así como bodegas, áreas de restauración y curaduría.

Servicios al público: Desde la fachada, entrada, módulo de información, sanitarios, áreas de descanso, cafetería, paquetería, accesos para minusválidos, auditorios, estacionamientos y bibliotecas.

Espacios de exposición: Son los destinados para las exposiciones permanentes y temporales.

Con base en lo anterior, se integraron los siguientes puntos: costos, horarios, teléfonos, sitio web y vía de contacto.

El otro aspecto que se incluyó fue infraestructura, instalaciones y mantenimiento, en este sentido se comenzó por ver en qué tipo de edificio está establecido el recinto museográfico puesto que el hecho de que un museo se ubique en un edificio histórico o se haya realizado específicamente para albergar alguna colección histórica, artística o arqueológica, tienen distintas perspectivas que no lo hacen mejor ni peor a uno u otro. Establecer un museo en un recinto histórico implica adaptar un uso completamente distinto al cual fue hecho previamente.
En lo que respecta a las instalaciones y mantenimiento, se contemplan, por un lado, factores internos como lo son el uso de las instalaciones por parte del personal de los museos y por parte del público visitante; y por el otro, factores externos como el clima y medio ambiente. En este último punto, se toma en consideración la variación climática en la que se encuentran ubicados los museos y la antigüedad de los edificios, pues las afectaciones son distintas, por lo que se incluyó determinar el tipo de afectación de cada uno. Para determinar el factor y deterioro, se utilizó como referencia el manual propuesto por la UNESCO (1969), La conservación de los bienes culturales (museos y monumentos, XI).

Finalmente, en este apartado, se incluyó el diagnóstico de los servicios brindados a los visitantes, debido a que, el tipo de edificio e instalaciones de cada recinto, influye en la calidad de los servicios y el impacto en lo que se ha denominado como "la experiencia del visitante".

Los servicios que se integran en el diagnóstico tienen que ver las necesidades básicas que todo espacio comercial, de entretenimiento y cultural debe tener y con el grado de accesibilidad del recinto con sus visitantes.

\section{Rasgos históricos y conformación de la colección}

Este rubro permite establecer la trayectoria de los museos en el estado y su cronología, la conformación de las colecciones y el tipo de acervo, evidencian la diversidad museológica de la entidad y con ello, poder establecer marcos comparativos entre los museos; además parte importante de la historia de los museos y de las colecciones arqueológicas que conforman las exhibiciones, se han conformado en gran medida por la investigación arqueológica veracruzana.

\section{Tipo de exposición y experiencia museográfica}

Para el diagnóstico de los museos en el es- 
tado, en este apartado solo tomaremos el tipo de exposición por su duración (permanentes, temporales o itinerantes) y por experiencia museográfica, porque nos permitieron saber qué tipo de mensaje comunican y transmiten a sus visitantes, y si en estos últimos surgen motivaciones, lazos de identidad y referencia con su patrimonio cultural.

Para el diseño de la ficha, retomamos la propuesta de Josep Ballart (2008: 6), con relación a la experiencia museográfica:

- Contemplativas: promueven el goce y la experiencia estética. Estimulan la apreciación individual de las obras de arte, los textos informativos son reducidos en número y extensión, y la visita se apoya en audioguías, hojas de sala, folletos y otras formas de interpretación que aportan una información básica.

- Contextuales o didácticas: son exposiciones temáticas, principalmente de historia o antropología, que pretenden contextualizar adecuadamente los objetos para descubrir su valor y significado, favoreciendo así su comprensión. Van acompañados de una gran cantidad de textos, dibujos, fotografías, planos y audiovisuales.

- Ambientales: pretenden contextualizar, pero recreando de manera conmovedora ambientes históricos, arqueológicos y etnográficos originales, donde los objetos o las réplicas ocupan su lugar original. Utiliza información textual mínima y pocos gráficos de apoyo.

- Interactivas: se basan en la manipulación de aparatos electrónicos o mecánicos para ejemplificar algún proceso o fenómeno científico. Utilizan algunas réplicas. Forman parte del modelo de los museos de ciencia y son una variante de las exposiciones didácticas, privilegian la interactividad manual y mental. Utilizan textos con instrucciones y explicaciones de los procesos generados.

\section{Servicios educativos y recursos didácticos}

Los servicios educativos de los museos, tie- nen como objetivos acercar, sensibilizar, promover y divulgar el conocimiento científico de las colecciones, además de motivar a la reflexión, el goce, el deleite y entretenimiento. Esta categoría tiene la finalidad de conocer cómo se están llevando a cabo las actividades, qué metodología siguen, qué valores patrimoniales promueven, a quiénes están dirigidas las actividades y los recursos con los que cuentan, o si existe algún impacto en la calidad de vida de los visitantes, todo ello mediante un servicio de calidad que se realiza a través de las visitas guiadas, talleres, materiales didácticos, cursos de verano y eventos culturales.

\section{Tipo de público}

Parte importante de la función de los museos es el público (real, potencial y no público). Aunque para definir su tipología, perfil, experiencia y percepción sobre la visita, existen diversas referencias teóricas y metodológicas para su estudio, para el diseño de la herramienta se toma en consideración para poder establecer si existe o no una relación con el tipo de exposición y experiencia museográfica. La gran mayoría de los museos no se diseñan para el "gran público" sino más bien, para ciertos sectores especializados según la colección.

\section{Registro de afluencia}

La afluencia se va a definir, como lo indica la Real Academia de la Lengua Española, como "acción y efecto de afluir", "abundancia", lo que quiere decir que es la concurrencia, el número de personas que acuden a determinado lugar, zona o sitio. Para el caso de la ficha de registro se contempla la afluencia estimada a los museos, la cual especifica la cantidad y tipo de visitantes que acuden anualmente a dichos recintos. La clasificación se integra por: anual, nacionales y extranjeros. Sin embargo, hay que hacer un señalamiento, puesto que las categorías anteriores no permiten especificar con claridad, los datos que puede arrojar un estudio de público más amplio y preciso. 


\section{Tipo y duración de la visita}

La visita, es una de las actividades primordiales que realiza el público en los museos, ésta puede ser mediante recorridos guiados, herramientas/recursos de apoyo como el audioguia o de manera particular, es decir, por cuenta propia. Sin embargo, la visita se ha constituido como el momento principal en el cual el público tiene su primer contacto con los objetos del museo, por lo que su forma y contenido, juegan un papel fundamental y la clave para que la experiencia de visita sea o no significativa. Para esta categoría, se tomaron en consideración el tipo de visita (guiada o libre) y la duración aproximada que cada recinto tiene. Sin embargo, se tiene contemplado integrar la visita mixta, para conocer la preferencia y establecer relaciones con los estudios de público, en los cuales se integran los motivos de la visita y si fue planeado o no, y que factores influyeron.

La información que se espera obtener por medio de este instrumento, permitirá conocer al museo desde la óptica de su concepción, es decir, para qué fue hecho, cuál ha sido su objetivo, qué discurso ofrece dentro de sus salas; los servicios con los que cuentan, porque a pesar de que los recintos son lugares de aprendizaje y deleite, también son instituciones encargadas de brindar un servicio al público que los visita.

Otros aspectos que considera esta herramienta es saber cómo está instalada la colección, si hay o no mantenimiento de las vitrinas, si la colección ha incrementado y debido a qué, si llevan un control de sus visitantes, qué tipo de visitantes tienen, además de los recursos educativos con los que cuentan, como los son materiales didácticos, apoyo y difusión de la colección permanente o temporal, recorridos guiados, programas de formación y capacitación, todo esto con la finalidad de conocer el museo desde dentro, es decir desde las políticas culturales, sociales, económicas y educativas que lo rigen, saber si estamos hablando de constantes o casos aislados, cuáles son sus diferencias y similitudes.

Finalmente, esta metodología es la base de la construcción de indicadores del observatorio, lo cual permitirá tener una sistematización de la información, elaboración y generación de productos geográficos y estadísticos para el estudio de los museos, y con ello, establecer políticas públicas encaminadas al desarrollo cultural de las comunidades.

\section{Referencias}

Ballart Hernández, J. (2008) Manual de museos, Madrid: Editorial Síntesis.

Bourdieu, P. \& Darvel, A. (2003). El amor al arte. Los museos europeos y su público. España: Paidós

Félix y Valenzuela, F. (2012). Gestión, planeación, diseño, producción y montaje de una exposición museográfica, en Cuadernos para la Gestión Cultural Municipal (2) 133-196.

Román Nieto, A. M. (2017). Entre Piedras y Vitrinas: el patrimonio museológico de Veracruz, en Proyecto ArKeopatías./ "Textos de la casa \#118? en línea. https:// arkeopatias.wordpress.com/

Santacana, J. \& Serrat, N. (Coord.). (2007) Museografía didáctica, España: Ariel.

UNESCO (1969). La conservación de los bienes culturales (museos y monumentos, XI). Francia: Organización de las Naciones Unidas para la Educación, la Ciencia y la Cultura.

Witker, R. (2001). Los museos, México: Tercer Milenio, CONACULTA.

Instituto Nacional de Estadística y Geografía http://www.inegi.org.mx/

Programa Nacional de Estudios de Público, INAH http://www.estudiosdepublico.inah.gob.mx/\#

Red de museos del INAH

http://www.inah.gob.mx/museos

-Registro de Museos Iberoamericanos (RMI)

http://www.rmiberoamericanos.org/

Sistema de Información Cultural (SIC)

http://sic.conaculta.gob.mx/lista.php?table=museo\&estado_id=30 